\title{
Focal White Matter Abnormalities Related to Neurocognitive Dysfunction: An Objective Diffusion Tensor Imaging Study of Children With Sturge-Weber Syndrome
}

\author{
BÁLINT ALKONYI, RAJKUMAR M. GOVINDAN, HARRY T. CHUGANI, MICHAEL E. BEHEN, JEONG-WON JEONG, \\ AND CSABA JUHÁSZ
}

\begin{abstract}
Departments of Pediatrics [B.A., R.M.G., H.T.C., M.E.B., J.-W.J., C.J.] and Neurology [H.T.C., M.E.B., C.J.], School of Medicine, Wayne State University, Detroit, Michigan, 48201; Translational Imaging Center [B.A., R.M.G., H.T.C., M.E.B., J.-W.J., C.J.], Children's
\end{abstract}

Hospital of Michigan, Detroit, Michigan, 48201

\begin{abstract}
White matter (WM) loss is associated with cognitive impairment in Sturge-Weber syndrome (SWS). In this study, we evaluated if cognitive and fine motor abnormalities are associated with impaired microstructural integrity in specific WM regions in SWS. Fifteen children with unilateral SWS (age: 3-12.4 y) and 11 controls (age: 6-12.8 y) underwent diffusion tensor imaging. Tractbased spatial statistics was used for objective comparisons of WM fractional anisotropy (FA) and mean diffusivity (MD) between the two groups. In the SWS group, WM FA and MD values were correlated with intelligence quotient (IQ) and fine motor scores, with age as a co-variate. Bilateral, multilobar WM areas showed decreased FA, whereas significant MD increases were confined to small ipsilateral posterior regions in SWS children. IQ in the SWS group (range: 47-128) was positively correlated with FA in the ipsilateral prefrontal WM and inversely associated with MD in the ipsilateral posterior parietal WM. A negative correlation between fine motor function and MD was found in ipsilateral frontal WM encompassing motor pathways. Microstructural WM abnormalities occur not only ipsilateral but also contralateral to the angioma in unilateral SWS. Nevertheless, cognitive and fine motor functions are related to diffusion abnormalities in specific ipsilateral, mostly frontal, WM regions. (Pediatr Res 69: 74-79, 2011)
\end{abstract}

S turge-Weber syndrome (SWS) is a neurocutaneous disorder associated with congenital facial port-wine stains, leptomeningeal angiomatosis, and glaucoma (1). Neurological manifestations, including motor deficit, visual field cut, progressive cognitive decline, and seizures, most often start during the first year of life and show a highly variable clinical course (2). Because intracranial pathology affects only one hemisphere in most cases (1,3), SWS is an excellent clinical model to study effects of early, unilateral brain damage on neurocognitive functions.

The pathophysiology of brain damage in SWS is poorly understood. Stasis and impaired blood drainage in abnormal pial veins may cause hypoxic-ischemic injury to the underlying cortex. Cortical abnormalities are common pathologic substrates of focal epilepsy and progressive neurocognitive

Received April 23, 2010; accepted August 16, 2010.

Correspondence: Csaba Juhász, M.D., Ph.D., Departments of Pediatrics and Neurology, Wayne State University School of Medicine, PET Center, Children's Hospital of Michigan, 3901 Beaubien Boulevard, Detroit, MI 48201; e-mail: juhasz@pet.wayne.edu

The study was supported by grant R01 NS041922 from the National Institute of Neurological Disorders and Stroke [C. J.]. impairments (4-6). In addition, the white matter (WM) may show structural and biochemical abnormalities, likely resulting from axonal and myelin injury (7-9). This can lead to a variable degree of WM atrophy in the affected hemisphere. Our previous studies demonstrated that the severity of subcortical WM atrophy, ipsilateral to the angioma, was a stronger predictor of cognitive functions than the degree of cortical atrophy (10). Further investigation of the WM is of high clinical relevance, because specific WM regions encompassing various corticocortical and corticosubcortical tracts play a crucial role in motor and neurocognitive functioning $(11,12)$.

Diffusion tensor imaging (DTI) is a sensitive noninvasive tool for studying the structural integrity of cerebral WM and can show abnormal microstructural abnormalities in WM even when they appear normal on conventional MRI. Tract-based spatial statistics (TBSS) provides an objective approach to study focal WM diffusion properties within/between groups (13-15). In this study, TBSS was used to objectively explore focal microstructural WM abnormalities associated with SWS. Two DTI parameters, fractional anisotropy (FA) — an indicator of WM coherence, axonal organization, and myelinationand mean diffusivity (MD) — the average magnitude of water diffusion-were analyzed.

This study had two main purposes: i) to determine which WM regions are commonly affected in children with unilateral SWS and ii) to identify specific WM areas whose diffusion properties are associated with cognitive abnormalities, fine motor functioning, or seizure variables.

\section{METHODS}

Subjects. Fifteen children (age range: 3-12.4 y; mean age: 7.2 y) with the diagnosis of SWS and unilateral hemispheric involvement were included in this study. All selected patients met the following inclusion criteria: 1) age at least $3 \mathrm{y}$; this age limit was selected to include only patients whose cognitive function could be assessed uniformly by the Wechsler intelligence scales and also to exclude very young patients with small brain volumes; 2) radiologic [MRI and/or 2-deoxy-2 $\left[{ }^{18}\right.$ F]fluoro-D-glucose PET (FDG-PET) $]$ evidence of unilateral brain involvement; 3) focal epilepsy and/or unilateral epileptiform

Abbreviations: DTI, diffusion tensor imaging; FA, fractional anisotropy; FDG, 2-deoxy-2 $\left[{ }^{18}\right.$ F $]$ fluoro-D-glucose; FSIQ, full-scale IQ; MD, mean diffusivity; MNI, Montreal Neurological Institute; SWS, Sturge-Weber syndrome; TBSS, tract-based spatial statistics; WM, white matter 
Table 1. Clinical and imaging data of the patients

\begin{tabular}{rccccl}
\hline Patient & $\begin{array}{c}\text { Age (y)/ } \\
\text { gender }\end{array}$ & $\begin{array}{c}\text { Duration of } \\
\text { epilepsy (y) }\end{array}$ & FSIQ & $\begin{array}{c}\text { Fine motor } \\
\text { function } \\
\text { scores }\end{array}$ & $\begin{array}{c}\text { Affected } \\
\text { brain } \\
\text { region }\end{array}$ \\
\hline 1 & $4.3 / \mathrm{M}$ & 3.9 & 75 & 29 & 1 FTPO \\
2 & $8.9 / \mathrm{M}$ & 7.1 & 76 & 10 & rTPO \\
3 & $11.3 / \mathrm{F}$ & 9.6 & 57 & 1 & rP \\
4 & $12.4 / \mathrm{M}$ & 10 & 47 & -9 & rFTPO \\
5 & $7 / \mathrm{M}$ & 6.5 & 47 & $-65^{*}$ & $1 \mathrm{FTPO}$ \\
6 & $3 / \mathrm{F}$ & 2.2 & 128 & 36 & rP \\
7 & $8.9 / \mathrm{M}$ & 8.6 & 82 & $-65^{*}$ & $1 \mathrm{~T}(\mathrm{P}) \mathrm{O}$ \\
8 & $8.1 / \mathrm{F}$ & 7.9 & 55 & $-65^{*}$ & $1 \mathrm{FTPO}$ \\
9 & $6 / \mathrm{F}$ & 5.7 & 60 & -64 & $1 \mathrm{FTPO}$ \\
10 & $4.5 / \mathrm{F}$ & 2.5 & 91 & 35 & $1(\mathrm{~T}) \mathrm{P}$ \\
11 & $9.5 / \mathrm{F}$ & 8.6 & 60 & $-65^{*}$ & rFTPO \\
12 & $6.3 / \mathrm{M}$ & 5.6 & 55 & 46 & rFTPO \\
13 & $4.1 / \mathrm{F}$ & 3.6 & 102 & 34 & $1 \mathrm{~T} \dagger$ \\
14 & $3.6 / \mathrm{F}$ & NA & 112 & 75 & $1 \mathrm{~T} \dagger$ \\
15 & $9.5 / \mathrm{M}$ & 8.9 & 93 & 23 & $1 \mathrm{PP}$ \\
\hline
\end{tabular}

* Fine motor function could not be assessed because of severe motor impairment in four subjects; the lowest score minus $1(-65)$ was assigned to them; only scores measured contralateral to the brain involvement are listed.

$\dagger$ These patients did not have leptomeningeal angioma; however, glucose PET showed left temporal hypometabolism and left facial angioma; intracranial involvement was further confirmed by the presence of clinical seizures or epileptiform discharges on scalp EEG.

NA, not applicable (this patient had only interictal spikes); M, male; F, female; 1, left; r, right; F, frontal; T, temporal; P, parietal; O, occipital. Letters in parenthesis indicate mild/partial involvement of the lobe.

EEG abnormalities; and 4) full-brain MRI scan with a good quality DTI sequence. Gadolinium-enhanced MRI revealed unilateral leptomeningeal angioma in all but two patients. Angiomas were located predominantly over the posterior cortex, also extending anteriorly in some cases. The side of hemispheric involvement was determined with interictal FDG-PET (based on cortical hypometabolism) in the two patients (patients 13 and 14 in Table 1, both with a unilateral facial angioma) without an identifiable leptomeningeal angioma. No apparent MRI or PET abnormalities could be seen in the contralateral hemisphere in any of the patients. Unequivocal involvement of the ipsilateral frontal lobe could be detected by clinical MRI and FDG-PET in seven children. Fourteen of the 15 patients had a history of clinical seizures (mean age of seizure onset: $11 \mathrm{mo}$; range: 2.5-29 mo; mean duration of epilepsy: $6.5 \mathrm{y}$; range: $2.5-9.6 \mathrm{y}$ ), while one patient (patient 14 ) had interictal spikes on scalp EEG without clinical seizures. Five patients had wellcontrolled epilepsy, eight patients had rare seizures (up to a few seizures per year), and only one patient (patient 5) had frequent (daily) seizures. Clinical and imaging data of the patients are presented in Table 1.

To compare the images of the patient group with those of normal controls, we used a healthy control group of 11 children (age range: 6-12.8 y; mean age: $10.5 \mathrm{y}$ ). All control children had a full-scale intelligence quotient (FSIQ) above 85 (above population mean - 1 SD) and had no current or historical medical, developmental, or psychiatric problems. Furthermore, they all had normal high-resolution brain MRI scans. The study was approved by the Institutional Review Board at Wayne State University, and written informed consent of the parent or legal guardian was obtained.

Neuropsychological evaluation. All children with SWS underwent a comprehensive neuropsychological assessment within $1 \mathrm{~d}$ of the MRI studies, performed by a pediatric neuropsychologist. Intellectual function was assessed using either the Wechsler Preschool and Primary Scales of Intelligence Scales for Children, Third Edition (WPPSI-III; age 36-87 mo) (16), or the Wechsler Intelligence Scales for Children, Third Edition (WISC-III; age $>87$ mo) (17). Global intellectual functioning was characterized by FSIQ. Children with SWS were also administered either the Purdue Pegboard task (18), if less than $5 \mathrm{y}$ of age, or the Grooved Pegboard task (19), if older than $5 \mathrm{y}$ of age. Raw manual dexterity scores ( $T$ scores) of the hand both contra- and ipsilateral to the affected hemisphere were calculated. In cases, where the tasks could not be performed due to severe motor impairment $(n=4$ and $n=1$ for the hands contralateral and ipsilateral to the affected hemisphere, respectively), the lowest score among the others minus 1 was assigned for the correlation analysis. Thus, the scores used for correlations ranged from -65 to 75 (median: 10). Manual dexterity scores of the hand ipsilateral to the affected hemisphere ranged from -136 to 59 (median: 36 ).
MRI data acquisition. MRI studies were performed on a Sonata 1.5T MR scanner (Siemens, Erlangen, Germany) using a standard head coil. During the scanning phase, SWS children younger than $7 \mathrm{y}$ of age were sedated. The MRI protocol for the patient group included an axial 3D gradient-echo T1weighted, an axial T2-weighted turbo spin-echo sequence, DTI, susceptibility weighted imaging (SWI), followed by a postgadolinium $(0.1 \mathrm{mmol} / \mathrm{kg})$ T1-weighted acquisition. Additional MR sequences included dynamic perfusion weighted imaging, magnetic resonance angiography and postgadolinium SWI, in some cases. DTI acquisition was performed using single-shot, diffusion-weighted, echoplanar imaging. The acquisition parameters included the following: $\mathrm{TR}=6600$; $\mathrm{TE}=97 \mathrm{~ms}$; $\mathrm{NEX}=8$; acquisition matrix $=$ $128 \times 128 ;$ bandwidth $=95 \mathrm{KHz} ; \mathrm{FOV}=230 \times 230 \times 3 \mathrm{~mm}^{3}$, voxel size $=$ $1.8 \times 1.8 \times 3 \mathrm{~mm}$ with $2 \mathrm{~b}$ values $\left(0,1000 \mathrm{~s} / \mathrm{mm}^{2}\right)$ applied sequentially in six noncollinear directions. The acquisition of DTI images was performed for both the patients and the DTI controls on the same scanner with the same protocol. In the control group, only diffusion tensor and volumetric T1weighted images were obtained.

Image analysis. All images were visually inspected and affine corrected for motion and other imaging artifacts. The spatial maps of DTI parameters were estimated using multivariate least squares fitting approach implemented in the DtiStudio software (www.mristudio.org) (20). Subsequently, the brain matter was extracted on the nondiffusion weighted (b0) images using the Brain Extraction Tool (BET) available with the FSL software (FMRIB Software Library, www.fmrib.ox.ac.uk/fsl) (21); the used fractional intensity threshold was 0.35 . The extracted brain masks were applied to both the FA and MD images, and these masked images were used for TBSS analysis (13). As six patients had right hemispheric brain involvement, the FA and MD images of these children were right-left flipped. Subgroups with left or right hemispheric involvement were not analyzed separately because of the limited number of cases. Importantly, the age, IQ, and motor function scores did not differ significantly between patients with left and right hemispheric involvement ( $p>0.1$ in all group comparisons, independent samples $t$ tests).

Statistical analysis was performed using TBSS (13), which is part of FSL. Details of the TBSS analysis have been described in our recent studies $(14,15)$. In brief, all FA images were aligned into a common space [Montreal Neurological Institute (MNI) 152 space] using nonlinear registration. An in-house created pediatric MNI FA template was used as a target for registration of individual FA data in this study. The transformed FA images of all participants were combined to create a group-specific mean FA image. This mean FA image was then thinned to create a mean skeleton image of the WM tracts. This mean skeleton was later applied to the registered FA images of individual participants. After the search for the maximum FA value along the width of the WM tract, perpendicular to the skeleton voxel, the maximum FA values were projected onto the skeleton voxel for further statistical analysis. An FA threshold of 0.25 was used to differentiate between gray matter (GM) and WM. In addition, using the FA image-derived skeleton, the maximum values along the direction perpendicular to the tract of the MD image were projected to a separate skeleton image.

Study design. Cross-subject statistical analysis of individual skeleton images of both controls and SWS children for both contrasts (controls $>$ SWS) and (controls $<$ SWS), with age as a covariate, was performed using nonparametric permutation tests (5000 permutations/design), as described previously (14), with a cluster size threshold of $>3$ and a $p$ value of $<0.01$ for significance, after correcting for multiple comparisons. The strict $p<0.01$ significance threshold was used because of the multiple design matrices to avoid false-positive results. Skeletal voxels significantly different between the groups were isolated, labeled, and expanded to include the full width of the WM tract, which was then used as a mask permitting calculation of the mean FA and MD values for the width of the tract for individual subjects. In addition, analysis of covariance (ANCOVA) was performed using PASW Statistics 18 to calculate age-adjusted mean FA and MD values of these regions for the patient and control group by factoring out the effect of age. This expansion procedure also facilitated better visualization of the identified voxel clusters. In addition, to address potential left-right asymmetries in normal brain that could potentially confound our group comparisons including flipped images, FA and MD images of all control subjects were left-right flipped, and these flipped images were compared with the original (nonflipped) image set, using the same parameter set and statistical threshold, which was used to compare the SWS and control groups. After repeating the initial steps of TBSS using only the images of the SWS group, separate general linear model design matrices were applied to seek for skeletal voxel clusters with FA/MD values correlating with FSIQ, fine motor function scores, age at seizure onset, and duration of epilepsy, after controlling for age (13). Correlations with $p<0.01$ were considered to be significant. A DTI atlas was used to determine which WM fiber tracts were involved in voxel clusters identified by each analysis (22). 
A

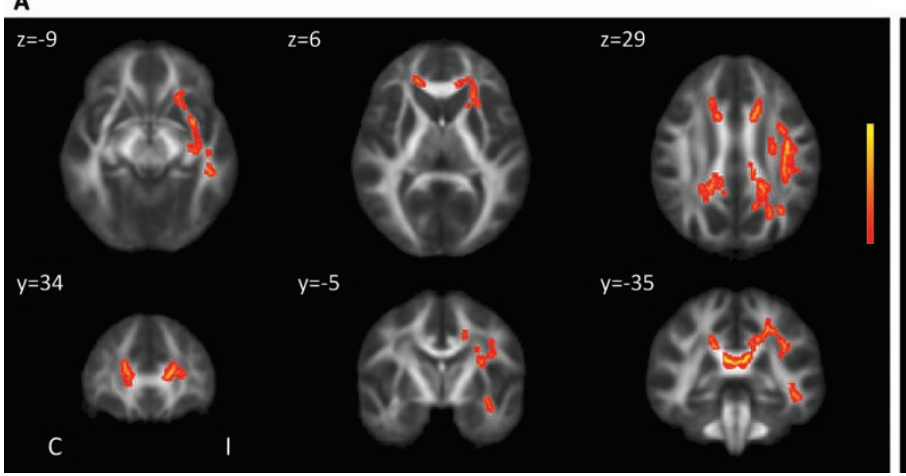

B

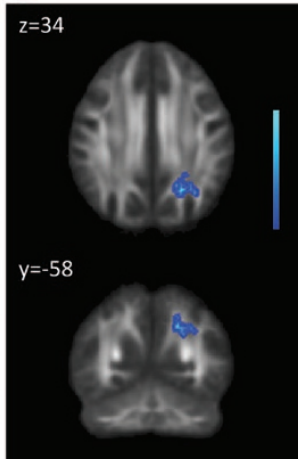

Figure 1. Tract-based comparison of WM FA $(A)$ and MD $(B)$ between children with SWS and healthy control children. To facilitate visualization, regions showing significant FA decrease/MD increase ( $p<0.01$, after correcting for multiple comparisons) are thickened using the tbss_fill script implemented in FSL. (A) The red/yellow voxels indicate WM areas where the FA was significantly reduced in patients compared with controls (after age differences between the two groups were factored out). (B) Blue-light blue voxels indicate brain regions where MD was significantly higher in patients compared with controls (after age factored out). Results are projected on the pediatric template FA image in MNI 152 space. Coordinates in MNI 152 space are indicated. I, side ipsilateral to the affected hemisphere; C, contralateral side. Output is color coded to enhance appearance of skeleton within final output. Brighter colors indicate voxels closer to the WM skeleton.

\section{RESULTS}

The comparison between the SWS and control group showed bilateral regions with significantly decreased FA in the SWS children (5.6 and 1.1\% of the mean WM skeleton of the brain in the ipsilateral and contralateral hemispheres, respectively), including ipsilateral, multilobar regions, and less extensive contralateral callosal and corona radiata areas (Fig. 1A). Age-adjusted mean FA of the abnormal, "expanded " skeleton voxels was $0.56(\mathrm{SD}=0.076)$, whereas that of the controls was 0.74 ( $\mathrm{SD}=0.091)$. Significant MD increases were, in contrast, much smaller and confined to ipsilateral posterior WM regions (posterior corona radiata; $0.31 \%$ of the mean WM skeleton; Fig. $1 B$ ). Age-adjusted mean MD of the abnormal expanded skeleton voxels was $0.882 \times 10^{-3} \mathrm{~mm}^{2} / \mathrm{s}$ $\left(\mathrm{SD}=5.24 \times 10^{-5}\right)$ compared with mean $\mathrm{MD}$ values of $0.765 \times 10^{-3} \mathrm{~mm}^{2} / \mathrm{s}\left(\mathrm{SD}=6.28 \times 10^{-5}\right)$ in the control group. Representative raw FA and MD images of a patient and a control subject are shown in Fig. 2. Most of the areas with increased MD also had significantly decreased FA. No voxel clusters showed increased FA or decreased MD values in the SWS group. Comparisons of the original and right-left flipped images of the control group showed no statistically significant differences between the two hemispheres for either FA and MD values.

Voxel clusters with FA values positively associated with FSIQ were identified in the ipsilateral frontal WM (total size: $0.35 \%$ of the mean WM skeleton; including callosal fibers, parts of the anterior thalamic radiation, anterior corona radiata, uncinate, and inferior fronto-occipital fascicles; Fig. $3 A$ ), indicating higher FA values in this area in children with better cognitive function. In contrast, areas with MD values inversely correlating with FSIQ were seen only in ipsilateral posterior regions $(0.32 \%$ of the mean WM skeleton; including parts of posterior thalamic radiation, inferior fronto-occipital, and inferior longitudinal fascicles; Fig. 3B).

Dexterity scores of the hand contralateral to the affected hemisphere inversely correlated with MD values in ipsilateral

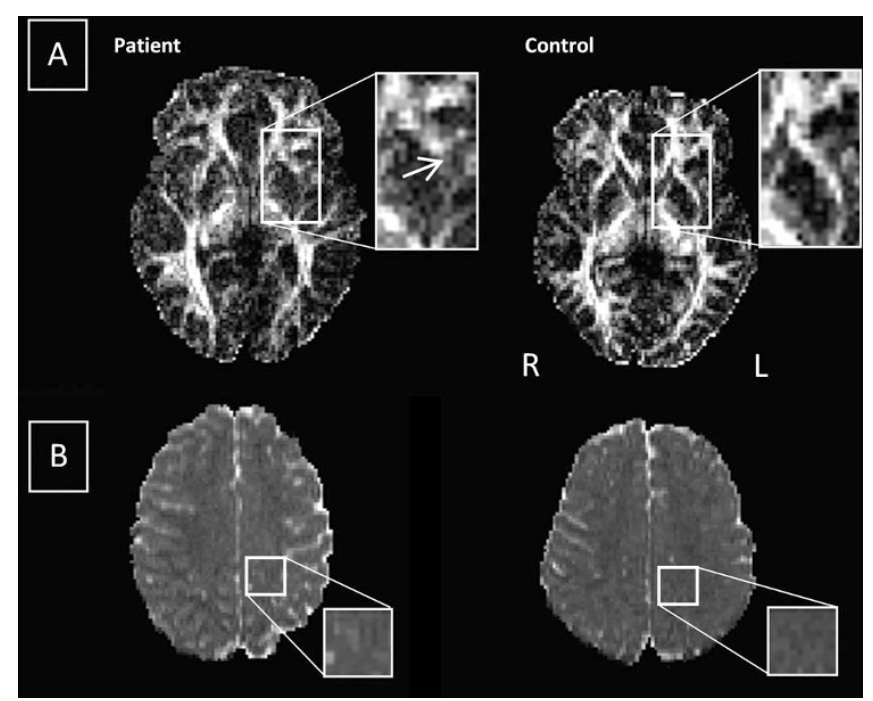

Figure 2. Representative axial FA $(A)$ and MD images $(B)$ of an 8.9-y-old patient (patient 7; left) with SWS and left hemispheric involvement (note the mild left posterior atrophy) and those of a control subject of the same age (right). Visual assessment of FA images can reveal areas suggestive of decreased FA (darker gray; arrow) in patients with SWS. MD differences are more subtle. L, left; R, right.

frontal WM encompassing parts of the corticospinal (CST) and corticopontine tracts (CPT), callosal fibers, superior longitudinal fascicle (SLF), and superior thalamic radiation (STR). Altogether $0.37 \%$ of the mean WM skeleton showed this correlation (Fig. 3C), indicating higher MD in this area in patients with worse hand motor function. In contrast, we found no areas with FA values either directly or inversely correlating with fine motor scores. In addition, no voxels could be identified with FA or MD values correlating with dexterity scores of the hand ipsilateral to the affected hemisphere. Finally, no voxel clusters with FA or MD values correlating with age at seizure onset or duration of epilepsy could be identified. 

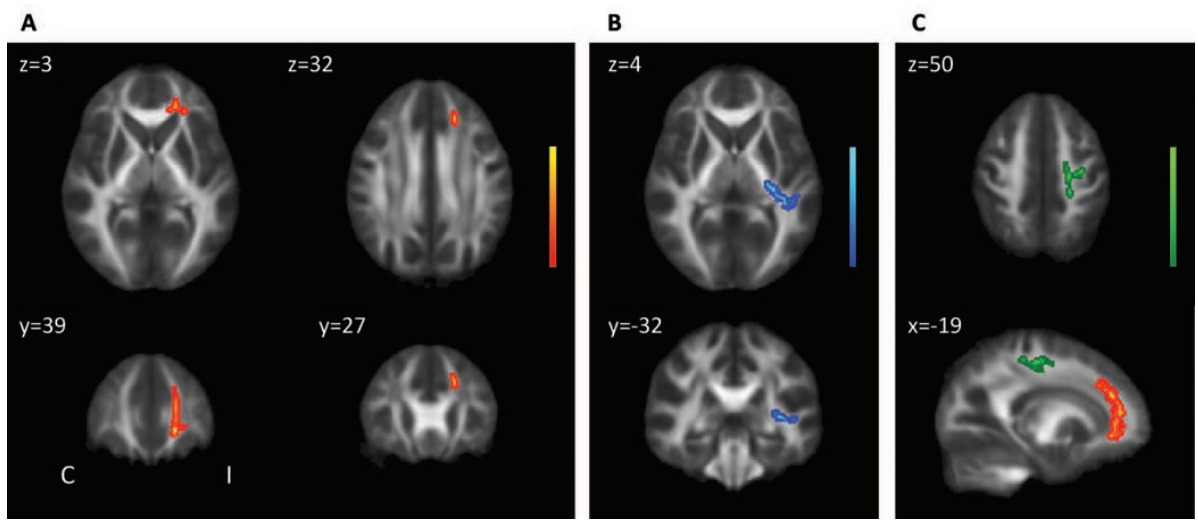

Figure 3. Results of correlations between DTI parameters and clinical variables in children with SWS. (A) Significant $(p<0.01)$ positive correlations between IQ and FA values in patients with SWS. The red/yellow voxels indicate WM regions where the FA positively correlated with IQ. (B) Significant inverse correlation between IQ and MD in patients with SWS. The blue/light blue voxels indicate WM areas where IQ inversely correlated with MD. (C) WM regions with a significant inverse association between fine motor scores of the hand (opposite to the affected hemisphere) and MD values (green/light green). On the sagittal image, the region with the positive correlation between IQ and FA is also shown (red/yellow) to demonstrate the locations of the two distinct WM regions correlating with IQ versus motor scores.

\section{DISCUSSION}

Recently, we reported that hemispheric WM volume loss ipsilateral to the leptomeningeal angioma is associated with poor cognitive function in children with unilateral SWS (10). This study builds on those findings by performing a tractbased analysis of WM diffusion parameters and identifying focal WM regions with microstructural impairment. Our results suggest that FA is the more sensitive DTI parameter showing extensive ipsilateral and smaller contralateral WM regions with decreased anisotropy compared with controls, whereas increased diffusivity (MD) was confined to the ipsilateral posterior WM, a general area most often abnormal on conventional MRI scans in SWS patients. Although conventional MRI and PET studies showed no frontal involvement in several patients $(n=7)$, multiple frontal $\mathrm{WM}$ areas showed decreased FA on the group level, suggesting common involvement of the frontal lobe WM. Concordant with this finding, a recent study on SWS subjects also found diffusion abnormalities in normally appearing WM (9). Our study also demonstrates that cognitive and fine motor dysfunctions are related to microstructural abnormalities in specific ipsilateral, but not contralateral, WM regions.

The exact mechanism leading to WM damage in SWS is not completely understood. Direct effects contributing to WM injury may include hypoxia due to impaired venous drainage; this is supported by observations of "accelerated myelination" in infants with SWS (23), and this may be followed by WM atrophy in older children (24). Cortical malformations and neuronal loss may also account for axonal loss and dysmyelination affecting diffusion parameters. Interestingly, some WM areas (including mostly callosal fibers) contralateral to the affected hemisphere also showed decreased FA, perhaps indicating Wallerian degeneration. This may be similar to findings from previous reports showing reduced anisotropy of the normalappearing CST remote from a cerebral infarct and interpreted as Wallerian degeneration (25). An alternative explanation could be the remote effect of the epilepsy itself (26).
Unilateral brain damage acquired early in life, as seen in SWS, may have an important impact on cognitive functions. Cortical injury in SWS plays a crucial role not only in cognitive but also in other neurological manifestations, such as seizures $(6,27)$. However, rapid progression of unilateral hemispheric demise in SWS may be associated with relatively preserved cognitive function, presumably because of more effective contralateral reorganization (4). As already mentioned, abnormal development or loss of WM may be a critical factor for cognitive decline in SWS (10). Our current data suggest that the degree of impairment of the (often normalappearing) frontal WM including anterior callosal fibers may be crucial in cognitive outcome. Previous research has shown that region-specific maturational trajectories in the developing brain coincide with corresponding functional development $(28,29)$. Furthermore, age-related microstructural degradation of certain brain regions is associated with different aspects of $\operatorname{cog}$ nitive decline (30). The observed correlation between IQ and DTI parameters in anterior and posterior WM may also be actual neuroanatomical substrates of distinct cognitive subdomains.

Not surprisingly, fine motor function of the hand contralateral to the affected hemisphere showed an inverse association with MD values of voxel clusters located in ipsilateral frontal WM encompassing callosal fibers, STR, CPT, CST, and SLF tracts. Concordantly, our previous tractography study evaluating the CST demonstrated increased MD values in SWS patients with more severe motor deficit (31). Lower $N$-acetylaspartate (NAA) levels in frontal lobe WM and GM are also associated with impaired motor function (8). Thus, this study along with our previous results underlines that dexterity in the affected hand highly depends on the severity of damage to frontal motor pathways in SWS.

The fact that we could not identify any WM voxel clusters with diffusion parameters correlating with the time of seizure onset or epilepsy duration suggest that epileptic activity is probably more associated with cortical pathology rather than WM damage. This notion is further supported by our previous 
MR-spectroscopy data showing that lower frontal lobe GM but not WM NAA was associated with earlier onset of seizures (8). The high metabolic demand of frequent seizures accompanied by an impairment of the cerebral hemodynamic response can worsen ischemic brain injury and lead to neurologic deterioration (32), especially in children with severe, intractable seizures. However, most of the children included in this study had relatively well-controlled seizures, with no significant impact on progressive brain damage, including WM damage.

Methodological considerations. This is a cross-sectional study with limited number of patients. The small sample size precluded the analysis of sex effects. Antiepileptic medication may also influence cognitive function, but the variety of drugs would make it hard to control for such effects. Importantly, the study sample was not homogenous with respect to the side of the hemispheric involvement. Therefore, to achieve reasonable statistical power for group analysis, the images of patients with right hemispheric involvement (smaller subgroup) were right-left flipped. As we know that WM hemispheric asymmetries exist in the developing brain (33), it could be argued that this step introduced potential confounding effects. However, a separate TBSS analysis of our control group (with the same parameters as for the patient analysis) did not show any significant left-right asymmetries in FA or MD, suggesting that this approach may not be sensitive to left-right flipping in relatively small study samples. Notably, we included only patients above the age of 3 y to obtain accurate, reliably comparable IQ scores measured by the Wechsler scales. However, even this selected patient group's age was lower than that of our control group. To minimize the potential confounding effect of brain development (34), age was included as a co-variate in all design matrices.

Although TBSS is an objective, automatic procedure, there are still some limitations that have been discussed in detail by the group who developed the software (13). Notably, abnormalities at tract junctions or crossings should be interpreted carefully. One can also argue that nonlinear registration of FA images of brains with regional atrophy to a template may not be accurate. However, the projection of individual subjects' aligned FA images onto the mean FA skeleton accounts for residual misalignments and makes the comparison of FA and MD values between groups reliable (13). Furthermore, as we applied general linear model analysis, which is designed to seek for linear associations, nonlinear correlations between DTI parameters and clinical variables might have been missed. Finally, the group comparison is designed to identify regions commonly affected in a group level and cannot indicate individual abnormalities.

\section{CONCLUSION}

In conclusion, our data demonstrate widespread WM diffusion abnormalities in patients with SWS suggesting significant microstructural brain tissue damage. WM abnormalities were present not only ipsilateral but also, to some extent, contralateral to the side of the apparent brain involvement. Nevertheless, cognitive functions appear to be associated with tissue damage of focal ipsilateral, but not contralateral, posterior and prefrontal WM. Dexterity of the hand opposite to the angioma correlates inversely with diffusivity of frontal areas encompassing motor pathways. Future longitudinal studies will be useful to evaluate whether early diffusion abnormalities in these WM regions have prognostic value for the clinical course of neurocognitive functions.

Acknowledgments. We thank Malek I. Makki, $\mathrm{PhD}$, and Yang Xuan, BS, for their technical support in the MRI acquisition. We also thank Majid Khalaf, MD, Anne Deboard, RN, and Jane Cornett, RN, for their assistance in sedation and the Sturge-Weber Foundation for referring patients to us and for families and children who participated in the study.

\section{REFERENCES}

1. Bodensteiner JB, Roach ES 1999 Sturge-Weber Syndrome. Sturge-Weber Foundation, Mt. Freedom, NJ, pp 1-10

2. Riela A, Roach E 2004 Sturge-Weber syndrome. In: Roach E, Miller V (eds) Neurocutaneous Disorders. Cambridge University Press, New York, NY, pp 179185

3. Pascual-Castroviejo I, Pascual-Pascual SI, Velazquez-Fragua R, Viano J 2008 Sturge-Weber syndrome: study of 55 patients. Can J Neurol Sci 35:301-307

4. Lee JS, Asano E, Muzik O, Chugani DC, Juhasz C, Pfund Z, Philip S, Behen M, Chugani HT 2001 Sturge-Weber syndrome: correlation between clinical course and FDG PET findings. Neurology 57:189-195

5. Juhasz C, Batista CE, Chugani DC, Muzik O, Chugani HT 2007 Evolution of cortical metabolic abnormalities and their clinical correlates in Sturge-Weber syndrome. Eur J Paediatr Neurol 11:277-284

6. Maton B, Krsek P, Jayakar P, Resnick T, Koehn M, Morrison G, Ragheb J, Castellano-Sanchez A, Duchowny M 2010 Medically intractable epilepsy in SturgeWeber syndrome is associated with cortical malformation: implications for surgical therapy. Epilepsia 51:257-267

7. Juhász C, Haacke EM, Hu J, Xuan Y, Makki M, Behen ME, Maqbool M, Muzik O, Chugani DC, Chugani HT 2007 Multimodality imaging of cortical and white matter abnormalities in Sturge-Weber syndrome. AJNR Am J Neuroradiol 28:900-906

8. Batista CE, Chugani HT, Hu J, Haacke EM, Behen ME, Helder EJ, Juhasz C 2008 Magnetic resonance spectroscopic imaging detects abnormalities in normalappearing frontal lobe of patients with Sturge-Weber syndrome. J Neuroimaging 18:306-313

9. Arulrajah S, Ertan G, M Comi A, Tekes A, Lin DL, Huisman TA 2010 MRI with diffusion-weighted imaging in children and young adults with simultaneous supraand infratentorial manifestations of Sturge-Weber syndrome. J Neuroradiol 37:51-59

10. Juhasz C, Lai C, Behen ME, Muzik O, Helder EJ, Chugani DC, Chugani HT 2007 White matter volume as a major predictor of cognitive function in Sturge-Weber syndrome. Arch Neurol 64:1169-1174

11. Riley JD, Franklin DL, Choi V, Kim RC, Binder DK, Cramer SC, Lin JJ 2010 Altered white matter integrity in temporal lobe epilepsy: association with cognitive and clinical profiles. Epilepsia 51:536-545

12. Voineskos AN, Rajji TK, Lobaugh NJ, Miranda D, Shenton ME, Kennedy JL, Pollock BG, Mulsant BH 2010 Age-related decline in white matter tract integrity and cognitive performance: a DTI tractography and structural equation modeling study. Neurobiol Aging, in press

13. Smith SM, Jenkinson M, Johansen-Berg H, Rueckert D, Nichols TE, Mackay CE Watkins KE, Ciccarelli O, Cader MZ, Matthews PM, Behrens TE 2006 Tract-based spatial statistics: voxelwise analysis of multi-subject diffusion data. Neuroimage 31:1487-1505

14. Govindan RM, Makki MI, Wilson BJ, Behen ME, Chugani HT 2010 Abnormal water diffusivity in corticostriatal projections in children with Tourette syndrome. Hum Brain Mapp, in press

15. Govindan RM, Behen ME, Helder E, Makki MI, Chugani HT 2010 Altered water diffusivity in cortical association tracts in children with early deprivation identified with Tract-Based Spatial Statistics (TBSS). Cereb Cortex 20:561-569

16. Wechsler D 2002 Wechsler Preschool and Primary Scale of Intelligence. 3rd ed. The Psychological Corporation, San Antonio, TX, pp 129-142

17. Wechsler D 1991 Manual for the Wechsler Intelligence Scale for Children. 3rd ed. The Psychological Corporation, San Antonio, TX, p 254

18. Tiffin J, Asher EJ 1948 The Purdue pegboard; norms and studies of reliability and validity. J Appl Psychol 32:234-247

19. Trites RL 1989 Lafayette Grooved Pegboard Task. Instruction/Owner's Manual. Lafayette Instrument Company, Lafayette, IN, pp 4-8

20. Jiang H, van Zijl PC, Kim J, Pearlson GD, Mori S 2006 DtiStudio: resource program for diffusion tensor computation and fiber bundle tracking. Comput Methods Programs Biomed 81:106-116

21. Smith SM, Jenkinson M, Woolrich MW, Beckmann CF, Behrens TE, Johansen-Berg H, Bannister PR, De Luca M, Drobnjak I, Flitney DE, Niazy RK, Saunders J, Vickers J, Zhang Y, De Stefano N, Brady JM, Matthews PM 2004 Advances in 
functional and structural MR image analysis and implementation as FSL. Neuroimage 23:S208-S219

22. Mori S, Wakana S, Van Zijl P 2005 MRI Atlas of Human White Matter. Elsevier B.V., Amsterdam, The Netherlands

23. Jacoby CG, Yuh WT, Afifi AK, Bell WE, Schelper RL, Sato Y 1987 Accelerated myelination in early Sturge-Weber syndrome demonstrated by MR imaging. J Comput Assist Tomogr 11:226-231

24. Pfund Z, Kagawa K, Juhasz C, Shen C, Lee JS, Chugani DC, Muzik O, Chugani HT 2003 Quantitative analysis of gray- and white-matter volumes and glucose metabolism in Sturge-Weber syndrome. J Child Neurol 18:119-126

25. Puig J, Pedraza S, Blasco G, Daunis IE, Prats A, Prados F, Boada I, Castellanos M, Sanchez-Gonzalez J, Remollo S, Laguillo G, Quiles AM, Gomez E, Serena J 2010 Wallerian degeneration in the corticospinal tract evaluated by diffusion tensor imaging correlates with motor deficit 30 days after middle cerebral artery ischemic stroke. AJNR Am J Neuroradiol 31:1324-1330

26. Concha L, Beaulieu C, Gross DW 2005 Bilateral limbic diffusion abnormalities in unilateral temporal lobe epilepsy. Ann Neurol 57:188-196

27. Comi AM 2003 Pathophysiology of Sturge-Weber syndrome. J Child Neurol 18:509-516

28. Gogtay N, Giedd JN, Lusk L, Hayashi KM, Greenstein D, Vaituzis AC, Nugent TF 3rd, Herman DH, Clasen LS, Toga AW, Rapoport JL, Thompson PM 2004 Dynamic mapping of human cortical development during childhood through early adulthood Proc Natl Acad Sci USA 101:8174-8179

29. Nagy Z, Westerberg H, Klingberg T 2004 Maturation of white matter is associated with the development of cognitive functions during childhood. J Cogn Neurosci 16:1227-1233

30. Kennedy KM, Raz N 2009 Aging white matter and cognition: differential effects of regional variations in diffusion properties on memory, executive functions, and speed. Neuropsychologia 47:916-927

31. Sivaswamy L, Rajamani K, Juhasz C, Maqbool M, Makki M, Chugani HT 2008 The corticospinal tract in Sturge-Weber syndrome: a diffusion tensor tractography study. Brain Dev 30:447-453

32. Aylett SE, Neville BG, Cross JH, Boyd S, Chong WK, Kirkham FJ 1999 SturgeWeber syndrome: cerebral haemodynamics during seizure activity. Dev Med Child Neurol 41:480-485

33. Wilde EA, McCauley SR, Chu Z, Hunter JV, Bigler ED, Yallampalli R, Wang ZJ, Hanten G, Li X, Ramos MA, Sabir SH, Vasquez AC, Menefee D, Levin HS 2009 Diffusion tensor imaging of hemispheric asymmetries in the developing brain. J Clin Exp Neuropsychol 31:205-218

34. Lebel C, Walker L, Leemans A, Phillips L, Beaulieu C 2008 Microstructural maturation of the human brain from childhood to adulthood. Neuroimage 40:1044-1055 